\title{
Research Article \\ Green's Function and Convergence of Fourier Series for Elliptic Differential Operators with Potential from Kato Space
}

\author{
Valery Serov \\ Department of Mathematical Sciences, University of Oulu, P.O. Box 3000, 90014 Oulu, Finland
}

Correspondence should be addressed to Valery Serov, vserov@cc.oulu.fi

Received 29 August 2009; Accepted 4 February 2010

Academic Editor: Martin D. Schechter

Copyright (C) 2010 Valery Serov. This is an open access article distributed under the Creative Commons Attribution License, which permits unrestricted use, distribution, and reproduction in any medium, provided the original work is properly cited.

We consider the Friedrichs self-adjoint extension for a differential operator $A$ of the form $A=$ $A_{0}+q(x)$, which is defined on a bounded domain $\Omega \subset \mathbb{R}^{n}, n \geq 1$ (for $n=1$ we assume that $\Omega=(a, b)$ is a finite interval). Here $A_{0}=A_{0}(x, D)$ is a formally self-adjoint and a uniformly elliptic differential operator of order $2 m$ with bounded smooth coefficients and a potential $q(x)$ is a realvalued integrable function satisfying the generalized Kato condition. Under these assumptions for the coefficients of $A$ and for positive $\lambda$ large enough we obtain the existence of Green's function for the operator $A+\lambda I$ and its estimates up to the boundary of $\Omega$. These estimates allow us to prove the absolute and uniform convergence up to the boundary of $\Omega$ of Fourier series in eigenfunctions of this operator. In particular, these results can be applied for the basis of the Fourier method which is usually used in practice for solving some equations of mathematical physics.

\section{Introduction}

Let $\Omega$ be a bounded domain in $\mathbb{R}^{n}(n \geq 1)$ with smooth boundary. We consider on $\Omega$ an elliptic differential operator of the form

$$
A=A_{0}(x, D)+q(x)
$$

where

$$
A_{0}(x, D)=\sum_{|\alpha| \leq 2 m} a_{\alpha}(x) D^{\alpha}
$$


is a formally self-adjoint differential operator of order $2 m, m=1,2, \ldots$. Here $D^{\alpha}=D_{1}^{\alpha_{1}} \cdot D_{2}^{\alpha_{2}}$. $\cdots D_{n}^{\alpha_{n}}$ with $D_{j}=-i\left(\partial / \partial x_{j}\right)$. The coefficients $a_{\alpha}(x)$ of the operator $A_{0}$ are assumed to be the complex-valued (in general) bounded smooth functions on the domain $\Omega$ for all $|\alpha| \leq 2 m$ such that $a_{\alpha}(x)$ are real valued for $|\alpha|=2 m$ and this operator $A_{0}$ satisfies the uniform ellipticity condition

$$
\sum_{|\alpha|=2 m} a_{\alpha}(x) \xi^{\alpha} \geq v|\xi|^{2 m}
$$

with some constant $v>0$, for all $x \in \Omega$ and all $\xi \in \mathbb{R}^{n}$. We assume that the potential $q(x)$ is a real-valued $L^{1}(\Omega)$-function satisfying the generalized Kato condition, that is,

$$
\sup _{x \in \Omega} \int_{\Omega}|q(y)| \omega_{n}(|x-y|) d y<\infty
$$

where function $\omega_{n}(t)$ for $t>0$ is defined by

$$
\omega_{n}(t)= \begin{cases}t^{2 m-n}, & 2 m<n \\ 1+|\log t|, & 2 m=n \\ 1, & 2 m>n .\end{cases}
$$

For $s>0$ we denote by $W_{2}^{s}(\Omega)$ the $L^{2}$-based Sobolev space, where $s$ indicates the "degree" of the smoothness; by $W_{2}(\Omega)$ we denote the closure of $C_{0}^{\infty}(\Omega)$ in $W_{2}^{s}(\Omega)$. We denote also by $B_{2, p}^{s}(\Omega), 1 \leq p \leq \infty$, Besov space (where $s$ indicates the smoothness) with the same notation for $\stackrel{\circ}{B}_{2, p}(\Omega)$ as for Sobolev space. The definition of Sobolev and Besov spaces as well as the embedding theorems for these spaces can be found in $[1,2]$.

Due to (1.4)-(1.5) the function $|q(x)|^{1 / 2}$ satisfies all conditions of Theorem 7.3 of [3] with $s=m$ and therefore for any $\delta>0$ we have the following inequality:

$$
\left|(q f, f)_{L^{2}(\Omega)}\right| \leq\left\||q|^{1 / 2} f\right\|_{L^{2}(\Omega)}^{2} \leq C_{0} N_{\delta}\left(|q|^{1 / 2}\right)\|f\|_{W_{2}^{m}(\Omega)}^{2}+C_{\delta} N_{1}\left(|q|^{1 / 2}\right)\|f\|_{L^{2}(\Omega)}^{2}
$$

where the constant $C_{0}$ depends only on $m$ and $n$, the constant $C_{\delta}$ depends only on $m, n$ and $\delta$, and the value $N_{\delta}\left(|q|^{1 / 2}\right)$ is defined by

$$
N_{\delta}\left(|q|^{1 / 2}\right):=\sup _{x \in \Omega} \int_{y \in \Omega,|x-y|<\delta}|q(y)| \omega_{n}(|x-y|) d y,
$$

where $\omega_{n}$ is as in (1.5).

Since the domain $\Omega$ is bounded then $N_{\delta}\left(|q|^{1 / 2}\right)$ tends to 0 as $\delta \rightarrow 0$. It immediately implies that there is a constant $C>0$ such that

$$
(A f, f)_{L^{2}(\Omega)} \geq \frac{v}{2}\|f\|_{W_{2}^{m}(\Omega)}^{2}-C\|f\|_{L^{2}(\Omega)}^{2}
$$


for all $f \in C_{0}^{\infty}(\Omega)$. Since $A_{\mu}=A+\mu I$ is positive for sufficiently large $\mu$ it has a positive self-adjoint Friedrichs extension $\left(A_{\mu}\right)_{F}$ such that

$$
\Phi\left(\left(A_{\mu}\right)_{F}\right) \subset \stackrel{\circ}{W}_{2}^{m}(\Omega)
$$

We define the Friedrichs extension of $A=A_{\mu}-\mu I$ to be $A_{F}=\left(A_{\mu}\right)_{F}-\mu I$ such that

$$
\boldsymbol{\nexists}\left(A_{F}\right) \subset \stackrel{\circ}{W}_{2}^{m}(\Omega)
$$

The domain of $A_{F}$ is given by

$$
\Phi\left(A_{F}\right)=\left\{f \in \stackrel{\circ}{W}_{2}^{m}(\Omega) \mid A f \in L^{2}(\Omega)\right\}
$$

It is also well known that this extension has a purely discrete spectrum $\left\{\lambda_{k}\right\}_{k=1}^{\infty}$ of finite multiplicity having the only one accumulation point at infinity $\left(\lambda_{k} \rightarrow+\infty\right)$ and a complete orthonormal system $\left\{u_{k}(x)\right\}_{k=1}^{\infty}$ of eigenfunctions in $L^{2}(\Omega)$.

To each function $f \in L^{2}(\Omega)$ we can assign the formal series

$$
f=\sum_{k=1}^{\infty} f_{k} u_{k}(x)
$$

where $f_{k}=\left(f, u_{k}\right)_{L^{2}(\Omega)}$ are the Fourier coefficients of $f$ with respect to the system $\left\{u_{k}(x)\right\}_{k=1}^{\infty}$.

The study of elliptic differential operators with smooth coefficients on a bounded domain $\Omega \subset \mathbb{R}^{n}$ with smooth boundary has a long history. We restrict the bibliographical remarks to the works that are of interest from the viewpoint of the present article.

The estimates for the Green's function and convergence of spectral expansions of a general elliptic differential operator of order $2 m$ with smooth coefficients on a bounded domain have been studied by many authors. We refer to a four-volume monograph of Hörmander [4, 5], the works of Alimov [6-9], Gårding [10], Krasovskiur [11, 12], Schechter [3] and others. We mention also the papers [13-15] of the author of the present which deal with the operators whose coefficients may have local singularities of specific order on an arbitrary smooth surface whose dimension is strictly less than that of the original domain. As to elliptic operators of order $2 m$ whose coefficients may have singularities in $L^{p}$, similar results have been mainly obtained for the Schrödinger operators $-\Delta+q(x)$ on $\mathbb{R}^{3}$ with $q$ from $L^{2}$ or in any dimensions but with $q$ which may have given singularity at one point. For such results, see Alimov and Joó [16], Ashurov [17], Ashurov and Faiziev [18], Khalmukhamedov $[19,20]$, Serov [21, 22], Serov and Buzurnyuk [23], and others. Some survey of resent results concerning $L^{p}$ theory of elliptic differential operators of order $2 m$ can be found in the articles of Davies [24, 25].

The aim of this paper is to prove the following results.

Theorem 1.1. Suppose that $q$ satisfies condition (1.4), then there exist constants $C>0, \delta>0$ and $\lambda_{0}>0$ such that for all $\lambda \geq \lambda_{0}$ the Green function $G(x, y, \lambda)$ of the operator $A_{F}+\lambda I$ exists and satisfies 
the following estimates:

(1) $2 m<n$, $|G(x, y, \lambda)| \leq C|x-y|^{2 m-n} e^{-\delta|x-y| \lambda^{1 / 2 m}}$,

(2) $2 m=n$, $|G(x, y, \lambda)| \leq C\left(1+\left|\log \left(|x-y| \lambda^{1 / 2 m}\right)\right|\right) e^{-\delta|x-y| \lambda^{1 / 2 m}}$,

(3) $2 m>n$, $|G(x, y, \lambda)| \leq C \lambda^{(n-2 m) / 2 m} e^{-\delta|x-y| \lambda^{1 / 2 m}}$

for all $x, y \in \Omega$ and $\lambda \geq \lambda_{0}$.

Without loss of generality, in the following theorem we assume that $A_{F}$ is positive.

Theorem 1.2. The Fourier series (1.12) converges absolutely and uniformly on the domain $\Omega$ for any function $f$ from the domain of the operator $A_{F}^{\sigma}$ for $\sigma>n / 4 m$.

One of the main results of the present paper is Theorem 1.1 which concerns the estimates up to the boundary of the domain $\Omega$ for the Green's function of an elliptic differential operator of order $2 m$ with singular potential from the generalized Kato space. In all previous publications, as far as we know, the estimates for the Green function are proved on an arbitrary compact subset from the domain $\Omega$ and for the case when the coefficients of operator are either smooth or have some special type of singularities.

Another main result of this paper is Theorem 1.2. It gives some sufficient conditions which provide the absolute convergence up to the boundary of $\Omega$ of Fourier series in eigenfunctions for functions from the domain of some power of this operator. In addition to Theorem 1.2, we would like to take into consideration Theorem 3.7 (see Section 3 of the paper) which is the generalization of the well-known result of Peetre (see [26]) to the operators with singular coefficients. It can be mentioned also here that in the scale of the spaces associated with some powers of our operator the results of Theorems 1.2 and 3.7 are sharp (see, e.g., [14]).

This paper is organized such that Theorem 1.1 is proved in Section 2 and Theorem 1.2 in Section 3. Some additional theorems about the absolute convergence of Fourier series are also proved in Section 3.

\section{Green's Function}

In this section we obtain the estimates for the Green's function of the operator $A_{F}+\lambda I$ when $\lambda$ is positive and sufficiently large.

Definition 2.1. For $\lambda>0$ and $y \in \Omega$, a locally integrable function $F(\cdot, y, \lambda)$ on $\Omega$ is called a fundamental solution for an operator $A+\lambda I$ if and only if

$$
(A+\lambda I) F(x, y, \lambda)=\delta(x-y) .
$$

Equation (2.1) holds in the sense of distributions, that is,

$$
\int_{\Omega} F(x, y, \lambda)\left(A_{0}^{\prime}+q(x)+\lambda I\right) \varphi(x) d x=\varphi(y)
$$


for all $\varphi \in C_{0}^{\infty}(\Omega)$, where

$$
A_{0}^{\prime} \cdot=\sum_{|\alpha| \leq 2 m}(-1)^{|\alpha|} D^{\alpha}\left(a_{\alpha}(x) \cdot\right)
$$

is the transpose of $A_{0}$.

We will use the following result.

Proposition 2.2. There exists $\lambda_{0}>0$ such that for any $\lambda \geq \lambda_{0}$, the differential operator $A_{0}+\lambda I$ has a fundamental solution $F_{0}(x, y, \lambda)$. Furthermore, for any multi-index $\alpha, 0 \leq|\alpha| \leq 2 m-1$, there are constants $C_{0}>0, \delta>0$ such that the following estimates hold:

(1) $2 m<n+|\alpha|$,

$$
\left|D^{\alpha} F_{0}(x, y, \lambda)\right| \leq C_{0}|x-y|^{2 m-n-|\alpha|} e^{-\delta|x-y| \lambda^{1 / 2 m}},
$$

(2) $2 m=n+|\alpha|$,

$$
\left|D^{\alpha} F_{0}(x, y, \lambda)\right| \leq C_{0}\left(1+\left|\log \left(|x-y| \lambda^{1 / 2 m}\right)\right|\right) e^{-\delta|x-y| \lambda^{1 / 2 m}},
$$

(3) $2 m>n+|\alpha|$,

$$
\left|D^{\alpha} F_{0}(x, y, \lambda)\right| \leq C_{0} \lambda^{(n+|\alpha|-2 m) / 2 m} e^{-\delta|x-y| \lambda^{1 / 2 m}},
$$

for all $x, y \in \Omega$ and $\lambda \geq \lambda_{0}$.

The proof of Proposition 2.2 can be found in [6].

We will look for the fundamental solution $F(x, y, \lambda)$ of the operator $A_{0}(x, D)+q(x)+\lambda I$, for $\lambda$ positive and large enough, as a solution of the integral equation

$$
F(x, y, \lambda)=F_{0}(x, y, \lambda)-\int_{\Omega} F_{0}(x, u, \lambda) q(u) F(u, y, \lambda) d u,
$$

where $F_{0}(x, y, \lambda)$ is the fundamental solution of the operator $A_{0}+\lambda I$. By Proposition 2.2, $F_{0}(\cdot, y, \lambda)$ exists and belongs (at least) to $L^{1}(\Omega)$ uniformly with respect to $y$ from $\Omega$.

We need the following lemma, which may have interest of its own right.

Lemma 2.3. Assume that $q$ satisfies condition (1.4), then there is $\lambda_{0}>0$ such that for all $\lambda \geq \lambda_{0}$ the fundamental solution $F(x, y, \lambda)$ exists as a solution of the integral equation (2.4) and satisfies the following estimates:

(1) $2 m<n$,

$$
|F(x, y, \lambda)| \leq C|x-y|^{2 m-n} e^{-(\delta / 2)|x-y| \lambda^{1 / 2 m}},
$$

(2) $2 m=n$,

$$
|F(x, y, \lambda)| \leq C\left(1+\left|\log \left(|x-y| \lambda^{1 / 2 m}\right)\right|\right) e^{-(\delta / 2)|x-y| \lambda^{1 / 2 m}},
$$

(3) $2 m>n$,

$$
|F(x, y, \lambda)| \leq C \lambda^{(n-2 m) / 2 m} e^{-(\delta / 2)|x-y| \lambda^{1 / 2 m}},
$$

with some positive constant $C$, where $\delta$ is as in Proposition 2.2 and $x, y \in \Omega$. 
Proof. We solve the integral equation (2.4) by iterations. For any $j \geq 1$, we denote

$$
F_{j}(x, y, \lambda)=-\int_{\Omega} F_{0}(x, u, \lambda) q(u) F_{j-1}(u, y, \lambda) d u .
$$

We will prove by induction that there is $\lambda_{0}>0$ such that for all $\lambda \geq \lambda_{0}$ and for each $j=0,1,2, \ldots$

$$
\left|F_{j}(x, y, \lambda)\right| \leq \frac{C_{0}}{2^{j}} V_{n}(|x-y|) e^{-(\delta / 2)|x-y| \lambda^{1 / 2 m}}
$$

where $x, y \in \Omega, C_{0}$ is as in the Proposition 2.2 and $V_{n}$ is defined as

$$
V_{n}(|x-y|)= \begin{cases}|x-y|^{2 m-n}, & 2 m<n, \\ 1+\left|\log \left(|x-y| \lambda^{1 / 2 m}\right)\right|, & 2 m=n \\ \lambda^{(n-2 m) / 2 m}, & 2 m>n .\end{cases}
$$

It is clear that for $j=0$ estimate (2.6) holds. And it is also clear that (2.6) holds for the case when $2 m>n$ for each $j=1,2, \ldots$, by choosing $\lambda_{0}$ large enough.

In the case $2 m \leq n$ (considering two possibilities $|x-u| \geq|u-y|$ and $|x-u| \leq|u-y|$ ) in order to prove (2.6) it is enough to prove that there exists $\lambda_{0}>0$ such that

$$
C_{0} \int_{\Omega} V_{n}(|x-u|)|q(u)| e^{-(\delta / 2)|x-u| \lambda^{1 / 2 m}} d u \leq \frac{1}{2}
$$

for all $x, y \in \Omega$ and $\lambda \geq \lambda_{0}$, where constant $C_{0}$ is as in Proposition 2.2.

Indeed, since for $2 m<n$ we have

$$
C_{0} \int_{\Omega} V_{n}(|x-u|)|q(u)| e^{-(\delta / 2)|x-u| \lambda^{1 / 2 m}} d u=C_{0} \int_{\Omega} \omega_{n}(|x-u|)|q(u)| e^{-(\delta / 2)|x-u| \lambda^{1 / 2 m}} d u
$$

where $\omega_{n}$ is as in (1.4), then we can estimate the left-hand side of (2.8) as follows. If $|q(u)|>R$ then the integrals in the latter equality tend to zero as $R \rightarrow \infty$. The reason is due to that condition (1.4) the measure of the set $\{u \in \Omega:|q(u)|>R\}$ tends to zero as $R \rightarrow \infty$. If $|q(u)|<R$ then this integral can be estimated by

$$
R \int_{\Omega} V_{n}(|x-u|) e^{-(\delta / 2)|x-u| \lambda^{1 / 2 m}} d u \leq C \frac{R}{\lambda^{\prime}}
$$

where some positive constant $C$ depends only on $\delta$ and dimension $n$. 
In the case $n=2 m$ (considering two possibility $|x-y| \leq \lambda^{-1 / 2 m}$ and $|x-y| \geq \lambda^{-1 / 2 m}$ ) it can be proved that

$$
\begin{aligned}
& C_{0} \int_{\Omega} V_{n}(|x-u|)|q(u)| e^{-(\delta / 2)|x-u| \lambda^{1 / 2 m}} d u \\
& \quad \leq C_{0}^{\prime}\left(\int_{\Omega} \omega_{n}(|x-u|)|q(u)| e^{-(\delta / 2)|x-u| \lambda^{1 / 2 m}} d u+\log \lambda \int_{\Omega}|q(u)| e^{-(\delta / 2)|x-u| \lambda^{1 / 2 m}} d u\right) .
\end{aligned}
$$

Then, instead of estimate (2.10) we obtain

$$
R \int_{\Omega} V_{n}(|x-u|) e^{-(\delta / 2)|x-u| \lambda^{1 / 2 m}} d u \leq C \frac{R \log \lambda}{\lambda} .
$$

Combining these two facts (including (2.10) and (2.12)), (2.8) and choosing appropriately $R$ (with respect to $\lambda$ ) we may conclude that inequality (2.6) is proved. Since the solution $F(x, y, \lambda)$ of the integral equation (2.4) is given by the series

$$
F(x, y, \lambda)=\sum_{j=0}^{\infty} F_{j}(x, y, \lambda)
$$

the estimates (2.6) prove also Lemma 2.3.

As a consequence of Lemma 2.3 and Proposition 2.2 we can obtain the estimates for the derivatives of order $|\alpha| \leq 2 m-1$ of the fundamental solution $F(x, y, \lambda)$. For the derivatives of $F(x, y, \lambda)$ we use the following representation:

$$
D_{x}^{\alpha} F(x, y, \lambda)=D_{x}^{\alpha} F_{0}(x, y, \lambda)-\int_{\Omega} D_{x}^{\alpha} F_{0}(x, u, \lambda) q(u) F(u, y, \lambda) d u
$$

The following corollary holds.

Corollary 2.4. Assume that $q$ satisfies the condition (1.4), then for the derivatives of the fundamental solution $F(x, y, \lambda)$ of order $|\alpha| \leq 2 m-1$ the following estimates hold:

(1) $2 m<n+|\alpha|$,

$$
\left|D_{x}^{\alpha} F(x, y, \lambda)\right| \leq C|x-y|^{2 m-n-|\alpha|} e^{-(\delta / 2)|x-y| \lambda^{1 / 2 m}},
$$

(2) $2 m=n+|\alpha|$,

$$
\left|D_{x}^{\alpha} F(x, y, \lambda)\right| \leq C\left(1+\left|\log \left(|x-y| \lambda^{1 / 2 m}\right)\right|\right) e^{-(\delta / 2)|x-y| \lambda^{1 / 2 m}},
$$

(3) $2 m>n+|\alpha|$,

$$
\left|D_{x}^{\alpha} F(x, y, \lambda)\right| \leq C \lambda^{(n+|\alpha|-2 m) / 2 m} e^{-(\delta / 2)|x-y| \lambda^{1 / 2 m}},
$$

for some constant $C>0$, for all $x, y \in \Omega$ and $\lambda \geq \lambda_{0}$ (where $\lambda_{0}$ and $\delta$ are as in Lemma 2.3). 
The proof of the corollary follows immediately from the integral representation for the derivatives of $F(x, y, \lambda)$, estimates for the derivatives of $F_{0}(x, y, \lambda)$ in Proposition 2.2, estimates for $F(x, y, \lambda)$ in Lemma 2.3, and estimates for the kernels with weak singularities.

Let us note that the fundamental solution $F(\cdot, y, \lambda)$, which was obtained in Lemma 2.3, belongs (at least) to $L^{1}(\Omega)$ uniformly with respect to $y$ from $\Omega$.

Now we are in the position to introduce the Green's function of the operator $A_{F}+\lambda I$. If $\lambda$ is sufficiently large then the operator $A_{F}+\lambda I$ is positive and its inverse

$$
\left(A_{F}+\lambda I\right)^{-1}: L^{2}(\Omega) \longrightarrow L^{2}(\Omega)
$$

is a bounded operator. It is also an integral operator with kernel denoted by $G(x, y, \lambda)$. If we use for this integral operator the symbol $\widehat{G}(\lambda)$ then we have

$$
\left(A_{F}+\lambda I\right) \hat{G}(\lambda)=I, \quad \widehat{G}(\lambda)\left(A_{F}+\lambda I\right)=I, \quad G(x, y, \lambda)=\overline{G(y, x, \lambda)} .
$$

Definition 2.5. The kernel $G(x, y, \lambda)$ of the integral operator $\widehat{G}(\lambda)$ is called the Green's function of the operator $A_{F}+\lambda I$.

Proof of Theorem 1.1. For $\tau>0$, let $\Omega^{\tau}$ and $\Omega^{\tau / 2}$ be compact sets, each of them having a smooth boundary, with $\Omega^{\tau} \subset \Omega^{\tau / 2} \subset \Omega$ such that

$$
\begin{gathered}
d\left(\Omega^{\tau}, \partial \Omega\right)=\tau, \quad d\left(\Omega^{\tau / 2}, \partial \Omega\right)=\frac{\tau}{2}, \\
d\left(\Omega^{\tau}, \partial \Omega^{\tau / 2}\right)=\frac{\tau}{2} .
\end{gathered}
$$

Here $d(X, Y)$ denotes the distance between the sets $X$ and $Y$.

Let $F(x, y, \lambda)$ be a fundamental solution of the operator $A_{F}+\lambda I$ for $x, y \in \Omega$ and $\lambda$ sufficiently large. We choose the function $x \in C_{0}^{\infty}(\Omega)$ such that

$$
x(x)= \begin{cases}1, & x \in \Omega^{\tau}, \\ 0, & x \in \Omega \backslash \Omega^{\tau / 2},\end{cases}
$$

and set

$$
E(x, y, \lambda)=x(x) F(x, y, \lambda)
$$

By this equation the function $E(x, y, \lambda)$ is well defined for all $x, y \in \Omega$. Clearly, $E(x, y, \lambda)=$ $F(x, y, \lambda)$ for $x \in \Omega^{\tau}, y \in \Omega$. We will show that $E(x, y, \lambda)$ is a parametrix for $A_{F}+\lambda I$. To prove this, let us introduce the function

$$
Q(x, y, \lambda):=G(x, y, \lambda)-E(x, y, \lambda)
$$


and corresponding integral operator with kernel $Q(x, y, \lambda)$

$$
\widehat{Q}(\lambda):=\widehat{G}(\lambda)-\widehat{E}(\lambda),
$$

where $\widehat{E}(\lambda)$ and $\widehat{G}(\lambda)$ are integral operators in $L^{2}(\Omega)$ with kernels $E(x, y, \lambda)$ and $G(x, y, \lambda)$, respectively. Then it follows from (2.20) that

$$
\left(A_{F}+\lambda I\right) \widehat{E}(\lambda)=I+\widehat{P_{1}}(\lambda)
$$

where

$$
\begin{gathered}
\widehat{P_{1}}(\lambda)=-\left(A_{F}+\lambda I\right) \widehat{Q}(\lambda), \\
\widehat{Q}(\lambda)=-\widehat{G}(\lambda) \widehat{P_{1}}(\lambda) .
\end{gathered}
$$

If we denote by $P_{1}(x, y, \lambda)$ the kernel of the integral operator $\widehat{P_{1}}(\lambda)$, then it follows from $(2.24)$ that for any $f \in L^{2}(\Omega)$,

$$
\widehat{Q}(\lambda) f(x)=-\int_{\Omega}\left(\int_{\Omega} G(x, u, \lambda) P_{1}(u, y, \lambda) d u\right) f(y) d y
$$

and the kernel $Q(x, y, \lambda)$ (see (2.20)) has the form

$$
Q(x, y, \lambda)=-\int_{\Omega} G(x, u, \lambda) P_{1}(u, y, \lambda) d u,
$$

where $x, y \in \Omega$. As a matter of fact we cannot characterize and estimate the kernel $P_{1}(x, y, \lambda)$ from (2.22) - (2.24). That is why we will proceed a little bit differently, as follows. Equality (2.19) implies that in the sense of distributions the following representation holds:

$$
\left(A_{x}(x, D)+\lambda I\right) E(x, y, \lambda)=\chi(x) \delta(x-y)+P(x, y, \lambda)
$$

where $x \in \Omega(y \in \Omega$ is considered here as a parameter) and $\lambda$ sufficiently large. The function $P(x, y, \lambda)$ in $(2.27)$ will be of the form

$$
P(x, y, \lambda)=\sum_{\alpha>0} \frac{D^{\alpha} x(x)}{\alpha !} A_{0}^{(\alpha)}(x, D) F(x, y, \lambda)
$$

with the differential operator $A_{0}^{(\alpha)}(x, D)$ having the symbol $A_{0}^{(\alpha)}(x,-i \xi)=\partial_{\xi}^{\alpha} A_{0}(x,-i \xi)$. It is the polynomial in $\xi \in \mathbb{R}^{n}$ of order $\leq 2 m-1$ and therefore the differential operators $A_{0}^{(\alpha)}(x, D)$ are of order $\leq 2 m-1$. This fact allows us to estimate the function $P(x, y, \lambda)$ (in comparison with $P_{1}$ ). Indeed, by the choice of $x, D^{\alpha} \chi \neq 0$ only on the set $\Omega^{\tau / 2} \backslash \Omega^{\tau}$ and therefore the 
representation (2.28) and Corollary 2.4 imply that the following estimate holds:

$$
|P(x, y, \lambda)| \leq C|x-y|^{1-n} e^{-(\delta / 2)|x-y| \lambda^{1 / 2 m}}
$$

for all $x, y \in \Omega$ and with $\delta>0$ as in Corollary 2.4.

Now we need the following lemma.

Lemma 2.6. For all $x, y \in \Omega$

$$
\chi(y) G(x, y, \lambda)=\chi(x) F(x, y, \lambda)-\int_{\Omega} G(x, u, \lambda) P(u, y, \lambda) d u
$$

where $P$ is as in (2.28) and $X$ is defined as in (2.19).

Proof. We can rewrite (2.27) in the operator form as

$$
\left(A_{F}+\lambda I\right) \widehat{E}(\lambda)=\chi I+\widehat{P}(\lambda)
$$

or (using (2.20))

$$
\widehat{P}(\lambda)=(1-\chi) I-\left(A_{F}+\lambda I\right) \widehat{Q}(\lambda) .
$$

The latter equation implies

$$
\widehat{Q}(\lambda)=\widehat{G}(\lambda)((1-x) I)-\widehat{G}(\lambda) \widehat{P}(\lambda),
$$

and therefore (using (2.20) again)

$$
\widehat{G}(\lambda)(x I)=\widehat{E}(\lambda)-\widehat{G}(\lambda) \widehat{P}(\lambda) .
$$

But this is equivalent to (2.30). Thus, this lemma is proved.

In order to finish the proof of Theorem 1.1 let us introduce new functions $\widetilde{F}$ and $\widetilde{G}$ which are obtained from $F$ and $G$ multiplying by

$$
e^{(\delta / 4)|x-y| \lambda^{1 / 2 m}} \begin{cases}|x-y|^{n-2 m}, & 2 m<n, \\ \left(1+\left|\log \left(|x-y| \lambda^{1 / 2 m}\right)\right|\right)^{-1}, & 2 m=n, \\ \lambda^{(2 m-n) / 2 m}, & 2 m>n,\end{cases}
$$


respectively, where $\delta$ is as in Corollary 2.4. Then (2.30) (see Lemma 2.6) and estimate (2.29) formally yield the following estimate (for simplicity let us consider here only the case $2 m<n$, the cases $2 m \geq n$ can be considered similarly)

$$
\begin{aligned}
\sup _{x, y \in \Omega}|X(y) \widetilde{G}(x, y, \lambda)| \leq & \sup _{x, y \in \Omega}|\tilde{F}(x, y, \lambda)|+\sup _{x, y \in \Omega}|\widetilde{G}(x, y, \lambda)| \\
& \quad \times \sup _{x, y \in \Omega}\left(|x-y|^{n-2 m} \int_{\Omega}|x-u|^{2 m-n}|u-y|^{1-n} e^{-(\delta / 4)|u-y| \lambda^{1 / 2 m}} d u\right) .
\end{aligned}
$$

Considering two possibilities $|x-u| \leq|u-y|$ and $|x-u| \geq|u-y|$ the value in the latter brackets can be estimated from above by

$$
C \int_{\Omega}|u-y|^{1-n} e^{-(\delta / 4)|u-y| \lambda^{1 / 2 m}} d u \leq \frac{C}{\lambda^{1 / 2 m}}
$$

This estimate allows us to get from (2.36) that

$$
\sup _{x, y \in \Omega}|\widetilde{G}(x, y, \lambda)| \leq \sup _{x, y \in \Omega}|\widetilde{F}(x, y, \lambda)|+\frac{C}{\lambda^{1 / 2 m}} \sup _{x, y \in \Omega}|\widetilde{G}(x, y, \lambda)|
$$

Since

$$
\sup _{x, y \in \Omega}|\tilde{F}(x, y, \lambda)|<\infty
$$

then for $\lambda$ large enough (2.38) yields

$$
\sup _{x, y \in \Omega}|\tilde{G}(x, y, \lambda)|<\infty .
$$

Thus, Theorem 1.1 is completely proved.

\section{Convergence of Fourier Series}

Without loss of generality, we assume in this section that $A_{F}$ is positive. Then by the J. von Neumann spectral theorem for $A_{F}+\mu I$, where $\mu \geq \lambda_{0}$ with $\lambda_{0}$ as in Theorem 1.1, the following representation holds:

$$
\left(A_{F}+\mu I\right)^{\sigma}=\int_{0}^{\infty}(\lambda+\mu)^{\sigma} d E_{\lambda}
$$


where $\sigma$ is real and $\left\{E_{\lambda}\right\}_{\lambda=0}^{\infty}$ is the spectral resolution corresponding to the self-adjoint operator $A_{F}$. The domain of the operator (3.1) is defined by

$$
D\left(A_{F}^{\sigma}\right)=\left\{f \in L^{2}(\Omega): \int_{0}^{\infty} \lambda^{2 \sigma} d\left(E_{\lambda} f, f\right)_{L^{2}(\Omega)}<\infty\right\}
$$

In our case (in the case of pure discrete spectrum), the spectral projector $E_{\lambda}$ has the form

$$
E_{\lambda} f(x)=\sum_{\lambda_{k}<\curlywedge} f_{k} u_{k}(x)
$$

where $f_{k}=\left(f, u_{k}\right)_{L^{2}(\Omega)}$ are the Fourier coefficients of $f$ with respect to the system $\left\{u_{k}(x)\right\}_{k=1}^{\infty}$. Hence relations (3.1) and (3.2) become

$$
\begin{gathered}
A_{F}^{\sigma} f(x)=\sum_{k=1}^{\infty} \lambda_{k}^{\sigma} f_{k} u_{k}(x), \\
D\left(A_{F}^{\sigma}\right)=\left\{f \in L^{2}(\Omega): \sum_{k=1}^{\infty} \lambda_{k}^{2 \sigma}\left|f_{k}\right|^{2}<\infty\right\} .
\end{gathered}
$$

In addition, we need a special representation of the negative fractional powers of $A_{F}$. If we assume that $0<\tau<1$ then using well-known properties of Euler beta-function, one can obtain

$$
\left(A_{F}+\mu I\right)^{-\tau}=\frac{\sin \tau \pi}{\pi} \int_{0}^{\infty} t^{-\tau} \widehat{G}(\mu+t) d t
$$

where $\widehat{G}(\mu+t)$ is the integral operator with kernel $G(x, y, \mu+t)$ from Section 2. This representation shows that operator (3.6) is also integral with kernel denoted by $G_{\tau}(x, y, \mu)$. Using Theorem 1.1 of present work and well-known technique (see, e.g., [6]) it is not so difficult to prove the following estimates

$$
\left|G_{\tau}(x, y, \mu)\right| \leq C e^{-(\delta / 2)|x-y| \mu^{1 / 2 m}} \begin{cases}|x-y|^{2 m \tau-n}, & 2 m \tau<n \\ 1+|\log | x-y||, & 2 m \tau=n \\ 1, & 2 m \tau>n\end{cases}
$$

where $x, y \in \Omega, \delta$ is as in Theorem 1.1 and the constant $C>0$ depends on $\Omega$.

The following main lemma holds.

Lemma 3.1. For any function $f \in L^{2}(\Omega)$ and for $\sigma>n / 4 m$

$$
\left\|\left(A_{F}+\mu I\right)^{-\sigma} f\right\|_{L^{\infty}(\Omega)} \leq C \mu^{n / 4 m-\sigma}\|f\|_{L^{2}(\Omega)^{\prime}}
$$

where $\mu \geq \lambda_{0}$ with $\lambda_{0}$ as in Theorem 1.1. 
Proof. For any $\sigma>n / 4 m$, we write

$$
\sigma=\tau_{1}+\tau+\cdots+\tau_{m}, \quad 0<\tau_{j}<1, j=1,2, \ldots, m
$$

and represent the operator $\left(A_{F}+\mu I\right)^{-\sigma}$ as the product

$$
\left(A_{F}+\mu I\right)^{-\sigma}=\left(A_{F}+\mu I\right)^{-\tau_{1}} \cdot\left(A_{F}+\mu I\right)^{-\tau_{2}} \cdot \ldots \cdot\left(A_{F}+\mu I\right)^{-\tau_{m}} .
$$

Then, by applying the estimates (3.7) and Lemma 1 in [3], we arrive at (3.8). This completes the proof.

Corollary 3.2. Assume that $\sigma>n / 4 m$. There is a constant $C>0$ depending only on $\Omega$, such that the estimate

$$
\sum_{k=1}^{\infty} \frac{\left|u_{k}(x)\right|^{2}}{\left(\lambda_{k}+\mu\right)^{2 \sigma}} \leq C \mu^{n / 2 m-2 \sigma}
$$

holds uniformly in $x \in \Omega$ and $\mu \geq \lambda_{0}$.

Proof. By the spectral theorem and relation (3.4), we can rewrite inequality (3.8) in the form

$$
\left|\sum_{k=1}^{\infty}\left(\lambda_{k}+\mu\right)^{-\sigma} f_{k} u_{k}(x)\right| \leq C \mu^{n / 4 m-\sigma}\left(\sum_{k=1}^{\infty}\left|f_{k}\right|^{2}\right)^{1 / 2},
$$

where $f_{k}$ are the Fourier coefficients of $f$ with respect to the system $\left\{u_{k}(x)\right\}_{k=1}^{\infty}$. Now inequality (3.11) follows by duality. The proof is complete.

Remark 3.3. The inequality (3.11) has an independent interest since it gives the "bundle" estimate of the eigenfunctions in the form

$$
\sum_{\lambda \leq \lambda_{k}<2 \lambda}\left|u_{k}(x)\right|^{2} \leq C \lambda^{n / 2 m}
$$

which holds uniformly in $x \in \Omega$ and $\lambda$ large enough. Indeed, from (3.12) we have

$$
\sum_{2 \lambda \leq \lambda_{k}+\mu<3 \lambda} \frac{\left|u_{k}(x)\right|^{2}}{\left(\lambda_{k}+\mu\right)^{2 \sigma}} \leq C \mu^{(n / 2 m)-2 \sigma}
$$

uniformly in $x \in \Omega$. If we chose now $\mu=\lambda \geq \lambda_{0}$ then one can immediately obtain (3.13).

Now we are ready to prove Theorem 1.2. 
Proof of Theorem 1.2. Using the representation (3.4), the inequality (3.11), and the CauchySchwarz-Bunyakovsk 2 inequality, we obtain

$$
\begin{aligned}
\sum_{k=1}^{\infty}\left|f_{k} u_{k}(x)\right| & \leq\left(\sum_{k=1}^{\infty}\left|f_{k}\right|^{2}\left(\lambda_{k}+\mu\right)^{2 \sigma}\right)^{1 / 2}\left(\sum_{k=1}^{\infty}\left|u_{k}(x)\right|^{2}\left(\lambda_{k}+\mu\right)^{-2 \sigma}\right)^{1 / 2} \\
& \leq C \mu^{n / 4 m-\sigma}\left(\sum_{k=1}^{\infty}\left|u_{k}(x)\right|^{2}\left(\lambda_{k}+\mu\right)^{2 \sigma}\right)^{1 / 2} \leq C\left(\sum_{k=1}^{\infty}\left|f_{k}\right|^{2} \lambda_{k}^{2 \sigma}\right)^{1 / 2}
\end{aligned}
$$

uniformly in $x \in \Omega$ and for any fixed $\mu \geq \lambda_{0}$. Now the desired assertion follows from (3.5). Theorem 1.2 is completely proved.

The estimate (3.13) allows us to obtain a bit more precise result than in Theorem 1.2. Namely, the following corollary holds.

Corollary 3.4. Assume that the function $f$ satisfies the condition

$$
\sum_{l=0}^{\infty}\left(\sum_{2^{l} \leq \lambda_{k}<2^{l+1}}\left|f_{k}\right|^{2} \lambda_{k}^{n / 2 m}\right)^{1 / 2}<\infty
$$

where $f_{k}=\left(f, u_{k}\right)_{L^{2}(\Omega)}$ are the Fourier coefficients of $f$ with respect to the system $\left\{u_{k}(x)\right\}_{k=1}^{\infty}$, then the Fourier series (1.12) converges absolutely and uniformly on $\Omega$.

Let us assume now that the potential $q(x)$ satisfies the conditions

$$
\begin{gathered}
q(x) \in L^{2}(\Omega), \quad 4 m>n, \\
q(x) \in L^{p}(\Omega), \quad p>2,4 m=n, \\
q(x) \in L^{n / 2 m}(\Omega), \quad 4 m<n,
\end{gathered}
$$

then it is not so difficult to see that for the case $4 m \geq n$ conditions (3.17) imply the condition (1.4). For the case $4 m<n$ this condition (3.17) for $q$ must be considered in addition to the condition (1.4). The following result is valid.

Theorem 3.5. Suppose that the potential $q(x)$ satisfies conditions (3.17), then for any function $f \in$ $\stackrel{\circ}{W}_{2}^{2 m}(\Omega)$,

$$
\lim _{\curlywedge \rightarrow+\infty}\left\|f-E_{\curlywedge} f\right\|_{W_{2}^{2 m}(\Omega)}=0,
$$

where $E_{\curlywedge}$ isgiven by (3.3). 
Proof. Using the Sobolev embedding theorem we easily conclude that conditions (3.17) imply the following inclusion:

$$
\stackrel{\circ}{W}_{2}^{2 m}(\Omega) \subset D\left(A_{F}\right) .
$$

And for any $f(x) \in{\stackrel{\circ}{W_{2}}}^{2 m}(\Omega)$ the following inequality holds:

$$
\left\|A_{F} f\right\|_{L^{2}(\Omega)} \leq C\|f\|_{W_{2}^{2 m}(\Omega)} .
$$

Moreover, we may assert that the operator $A_{F}+\mu I$ is invertible for $\mu$ large enough. Indeed, since the function

$$
h(x):=\left(\left(A_{0}\right)_{F}+\mu I+q(x)\right) f(x), \quad f(x) \in{\stackrel{\circ}{W_{2}^{2 m}}}^{2 m}(\Omega),
$$

belongs to $L^{2}(\Omega)$, we have the representation for $f(x)$

$$
f(x)=-\left(\left(A_{0}\right)_{F}+\mu I\right)^{-1}(q f)(x)+\left(\left(A_{0}\right)_{F}+\mu I\right)^{-1}(h)(x),
$$

where $\left(A_{0}\right)_{F}+\mu I$ denotes the Friedrichs self-adjoint extension for $A_{0}+\mu I$ in $L^{2}(\Omega)$. Using again the Sobolev embedding theorem and conditions (3.17) we may conclude that the functions $h$ and $q f$ belong to $L^{2}(\Omega)$. The results of [6] yield that the operator $\left(A_{0}\right)_{F}+\mu I$ is invertible with small norm for its inverse operator (if $\mu$ is large enough). This fact and the latter equality imply that for $\mu$ large enough the operator $A_{F}+\mu I$ is also invertible and for any $h(x) \in L^{2}(\Omega)$ we have the following inequality:

$$
\left\|\left(A_{F}+\mu I\right)^{-1} h\right\|_{W_{2}^{2 m}(\Omega)} \leq C\|h\|_{L^{2}(\Omega)} .
$$

Now let $f(x) \in \stackrel{\circ}{W}_{2}^{2 m}(\Omega)$. Then (3.23) implies

$$
\begin{aligned}
\left\|f-E_{\lambda} f\right\|_{W_{2}^{2 m}(\Omega)} & =\left\|\left(A_{F}+\mu I\right)^{-1}\left(\left(A_{F}+\mu I\right) f-E_{\lambda}\left(A_{F}+\mu I\right) f\right)\right\|_{W_{2}^{2 m}(\Omega)} \\
& \leq C\left\|h-E_{\lambda} h\right\|_{L^{2}(\Omega)} \longrightarrow 0, \quad \lambda \longrightarrow+\infty
\end{aligned}
$$

where $h(x)=\left(A_{F}+\mu I\right) f(x)$ belongs to $L^{2}(\Omega)$ and the convergence to zero in the last term follows from the J. von Neumann spectral theorem. The proof is complete.

The Sobolev embedding theorem gives the immediate corollary. 
Corollary 3.6. Let $4 m>n$. Then for any $f(x) \in \stackrel{\circ}{\stackrel{s}{W}_{2}}(\Omega)$ with $s>n / 2$

$$
\lim _{\lambda \rightarrow+\infty} E_{\curlywedge} f(x)=f(x)
$$

holds uniformly in $x \in \Omega$.

The next theorem gives us some sufficient conditions which provide the absolute and uniform convergence of Fourier series (1.12) in the classical Besov and Sobolev spaces. Following [2], we use the symbol $\widetilde{B}_{2, p}^{s}(\Omega)=\left\{f: f \in B_{2, p}^{s}\left(R^{n}\right)\right.$, supp $\left.f \subset \bar{\Omega}\right\}$.

Theorem 3.7. Assume that the potential $q(x)$ belongs to Sobolev space $W_{2}^{2 m l}(\Omega)$, where $l=[n / 4 m]$ is an entire part of $n / 4 m$, then for any function $f$ from Besov space $\widetilde{B}_{2,1}^{n / 2}(\Omega)$ the Fourier series $(1.12)$ converges absolutely and uniformly on the domain $\Omega$.

Proof. Using the Sobolev embedding theorem and the following representation:

$$
A_{F}^{l+1} f(x)=\sum_{r=0}^{l+1} C_{r, l}\left(A_{0}\right)_{F}^{r}\left(q^{l+1-r}(x) f(x)\right)
$$

where $C_{r, l}$ are some constants, we can conclude that the condition for the potential $q(x)$ implies the following inclusion:

$$
\stackrel{\circ}{W}_{2}^{2 m(l+1)}(\Omega) \subset D\left(A_{F}^{l+1}\right) .
$$

Then using the results of [2] (see Theorem 4.3.2/1) we may conclude that

$$
\widetilde{B}_{2,2}^{2 m(l+1)}(\Omega) \subset \stackrel{\circ}{W}_{2}^{2 m(l+1)}(\Omega) \subset D\left(A_{F}^{(l+1)}\right) .
$$

Consequently, by Theorem 4.3.2/2 of [2] and by Peetre's method of real interpolation (see, e.g., [2]), we have

$$
\widetilde{B}_{2,1}^{n / 2}(\Omega)=\left(L^{2}(\Omega), \widetilde{B}_{2,2}^{2 m(l+1)}(\Omega)\right)_{n / 4 m(l+1), 1} \subset\left(L^{2}(\Omega), D\left(A_{F}^{(l+1)}\right)\right)_{n / 4 m(l+1), 1} .
$$

But the latter space is the interpolation space $D^{n / 4 m, 1}$ of Peetre (see [26]) for the elliptic differential operator of order $2 \mathrm{~m}$. Since estimate (3.13) for the spectral function holds in our case, we can apply the results of [26] and conclude that the proof of this theorem is complete.

Remark 3.8. If $n$ is even then the statement of this theorem holds for any function $f(x)$ from Besov space $\stackrel{\circ}{B}_{2,1}^{n / 2}(\Omega)$ due to the equality (see Theorem $4.3 .2 / 1$ of [2])

$$
\widetilde{B}_{2,1}^{n / 2}(\Omega)=\stackrel{\circ n / 2}{B_{2,1}}(\Omega) .
$$


And the Sobolev embedding theorem for Besov spaces (see, e.g., [2]) implies that this the-

orem also holds for any function $f(x)$ from Sobolev space $\stackrel{\circ}{W_{2}^{s}}(\Omega)$ with $s>n / 2$ and arbitrary integer $n$.

\section{Acknowledgment}

This work was supported by the Academy of Finland (application no. 213476, Finnish Programme for Centres of Excellence in Research 2006-2011).

\section{References}

[1] R. A. Adams and J. F. Fournier, Sobolev Spaces, Academic Press, New York, NY, USA, 2nd edition, 2003.

[2] H. Triebel, Interpolation Theory Function Spaces Differential Operators, Mir, Moscow, Russia, 1980.

[3] M. Schechter, Spectra of Partial Differential Operators, vol. 1, North-Holland, Amsterdam, The Netherlands, 1971, North-Holland Series in Applied Mathematics and Mechanics.

[4] L. Hörmander, The Analysis of Linear Partial Differential Equations, Vols. 1-2, Springer, New York, NY, USA, 1983.

[5] L. Hörmander, The Analysis of Linear Partial Differential Equations, Vols. 3-4, Springer, New York, NY, USA, 1985.

[6] S. A. Alimov, "Fractional powers of elliptic operators and isomorphism of classes of differentiable functions," Differentsial'nye Uravneniya, vol. 8, pp. 1609-1626, 1972 (Russian).

[7] S. A. Alimov, "Uniform convergence and summability of the spectral expansions of functions from $L_{p}^{\alpha}, "$ Differentsial'nye Uravneniya, vol. 9, no. 4, pp. 669-681, 1973.

[8] S. A. Alimov, "The spectral expansions of functions belonging to $H_{p}^{\alpha}$," Matematicheskii Sbornik, vol. 101(143), no. 1, pp. 3-20, 1976.

[9] S. A. Alimov, "On the absolute convergence of spectral expansions," Doklady Akademii Nauk, vol. 342, no. 4, pp. 446-448, 1995 (Russian).

[10] L. Gårding, "Dirichlet's problem for linear elliptic partial differential equations," Mathematica Scandinavica, vol. 1, pp. 55-72, 1953.

[11] J. P. Krasovskiǔ, "Isolation of the singularity in Green's function," Izvestiya Akademii Nauk SSSR. Seriya Matematicheskaya, vol. 31, pp. 977-1010, 1967 (Russian).

[12] J. P. Krasovski1̌, "Properties of Green's functions, and generalized solutions of elliptic boundary value problems," Doklady Akademii Nauk SSSR, vol. 184, no. 2, pp. 270-273, 1969.

[13] V.S. Serov, "On the fundamental solution of a differential operator with a singularity," Differentsial'nye Uravneniya, vol. 23, no. 3, pp. 531-534, 1987.

[14] V. S. Serov, "The absolute convergence of spectral expansions of operators with a singularity," Differentsial'nye Uravneniya, vol. 28, no. 1, pp. 127-136, 1992.

[15] V. S. Serov, "On spectral expansions of functions in $H_{p}^{\alpha}$ for a differential operator with a singularity on the surface," Doklady Rossiǔskaya Akademiya Nauk, vol. 340, no. 1, pp. 26-28, 1995.

[16] S. A. Alimov and I. Jo6, "On the Riesz summability of eigenfunction expansions," Acta Scientiarum Mathematicarum, vol. 45, no. 1-4, pp. 5-18, 1983.

[17] R. R. Ashurov, "Asymptotic behavior of a spectral function of the Schrödinger operator with potential $q \in L^{2}\left(\mathbb{R}^{3}\right)$," Differentsial'nye Uravneniya, vol. 23, no. 1, pp. 169-172, 1987.

[18] R. R. Ashurov and J. E. Faiziev, "On eigenfunction expansions associated with the Schrödinger operator with a singular potential," Differentsial'nye Uravneniya, vol. 41, no. 2, pp. 241-249, 2005.

[19] A. R. Khalmukhamedov, "Eigenfunction expansions for the Schrödinger operator with singular potentials," Differentsial'nye Uravneniya, vol. 20, no. 9, pp. 1642-1645, 1984.

[20] A. R. Khalmukhamedov, "Convergence of spectral expansion for a singular operator," Differentsial'nye Uravneniya, vol. 22, no. 12, pp. 2107-2117, 1986.

[21] V. S. Serov, "On the convergence of Fourier series in eigenfunctions of the Schrödinger operator with Kato potential," Matematicheskie Zametki, vol. 67, no. 5, pp. 755-763, 2000.

[22] V. S. Serov, "Fundamental solution and Fourier series in eigenfunctions of degenerate elliptic operator," Journal of Mathematical Analysis and Applications, vol. 329, no. 1, pp. 132-144, 2007. 
[23] N. S. Buzurnyuk and V. S. Serov, "On the convergence of Riesz means of spectral expansions that correspond to the Schrödinger operator with a singular potential," Differentsial'nye Uravneniya, vol. 32, no. 1 , pp. 83-89, 1996.

[24] E. B. Davies, Spectral Theory and Differential Operators, vol. 42 of Cambridge Studies in Advanced Mathematics, Cambridge University Press, Cambridge, UK, 1995.

[25] E. B. Davies, " $L^{p}$ spectral theory of higher-order elliptic differential operators," The Bulletin of the London Mathematical Society, vol. 29, no. 5, pp. 513-546, 1997.

[26] J. Peetre, "Absolute convergence of eigenfunction expansions," Mathematische Annalen, vol. 169, pp. 307-314, 1967. 


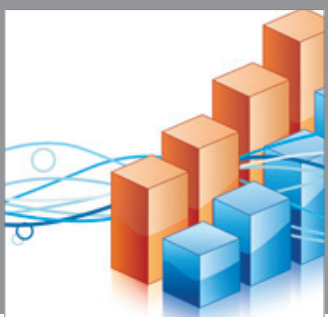

Advances in

Operations Research

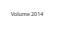

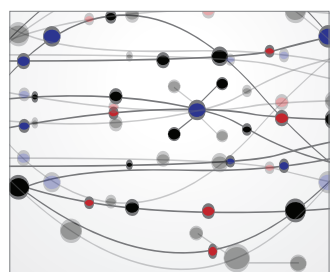

\section{The Scientific} World Journal
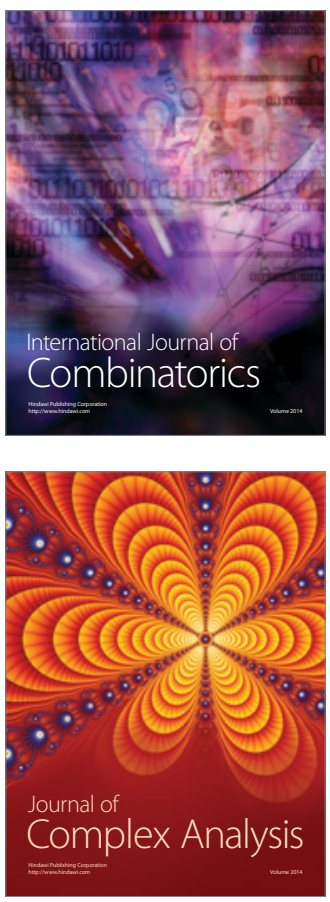

International Journal of

Mathematics and

Mathematical

Sciences
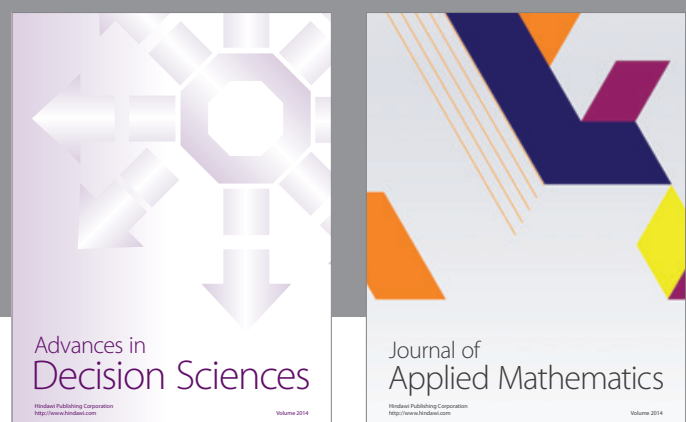

Journal of

Applied Mathematics
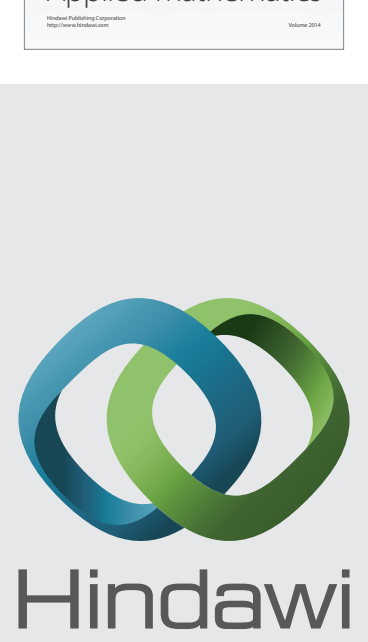

Submit your manuscripts at http://www.hindawi.com
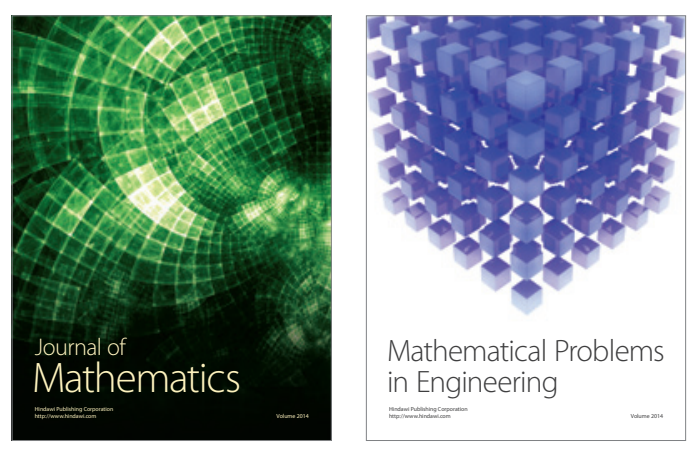

Mathematical Problems in Engineering
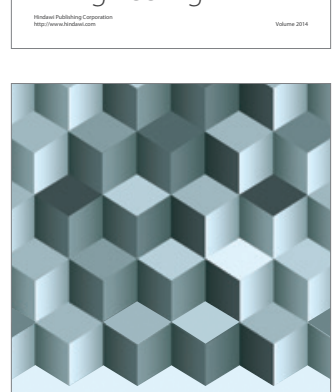

Journal of

Function Spaces
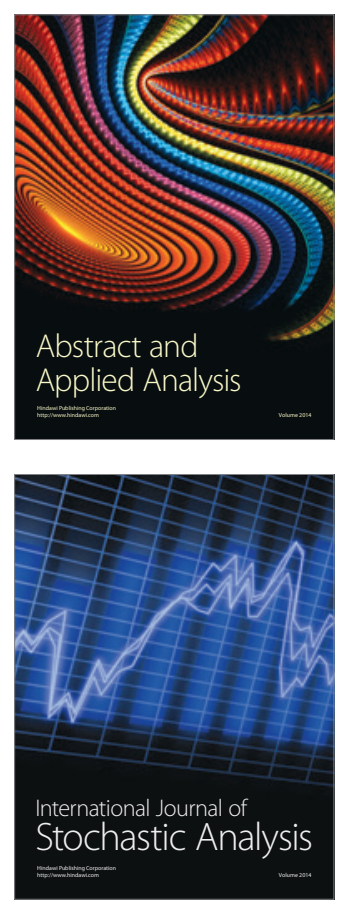

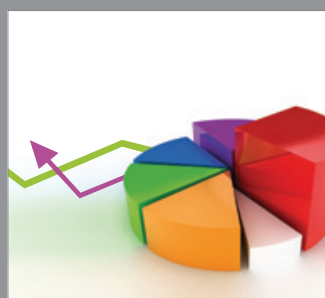

ournal of

Probability and Statistics

Promensencen
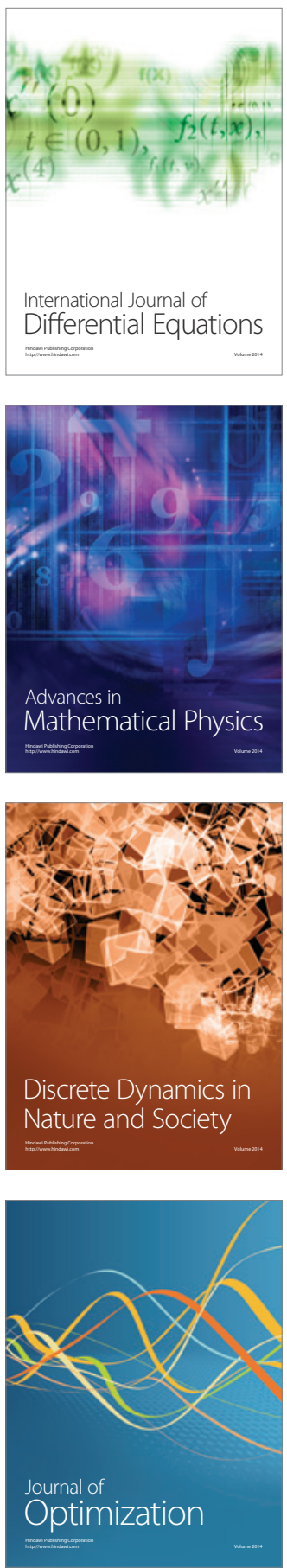